\title{
Numerical analysis of influence on gas supply hole number and radial clearance for the characteristics of hydroinertia gas journal bearings
}

\author{
Tomohiko Ise ${ }^{1}$, Sayuri Nakano ${ }^{1}$, Toshihiko Asami ${ }^{1}$, Yuki Endo ${ }^{2}$, Itsuro Honda ${ }^{1}$ \\ ${ }^{1}$ Graduate School of Mechanical Engineering, University of Hyogo, 2167 Shosha, Himeji, Hyogo 671-2280, Japan \\ ${ }^{2}$ JEOL RESONANCE Inc., 1-2, Musashino 3-Chome, Akishima, Tokyo 196-8558, Japan
}

\section{Email address:}

ise@eng.u-hyogo.ac.jp (T. Ise)

\section{To cite this article:}

Tomohiko Ise, Sayuri Nakano, Toshihiko Asami, Yuki Endo, Itsuro Honda. Numerical Analysis of Influence on Gas Supply Hole Number and Radial Clearance for the Characteristics of Hydroinertia Gas Journal Bearings. International Journal of Mechanical Engineering and Applications. Vol. 3, No. 1, 2015, pp. 16-21. doi: 10.11648/j.ijmea.20150301.13

\begin{abstract}
Hydroinertia gas journal bearings have been applied to solid state nuclear magnetic resonance spectroscopy. These bearings, which require a larger radial clearance than conventional externally pressurized bearings, achieve stable operation for ultra high-speed rotation of spinner shaft at several million rpm. However, their small diameters, which range within 1-6 mm, have limited experimental verifications of their static characteristics. A computational fluid dynamics analysis was therefore performed to fill this gap. The effects of radial clearance and gas supply hole number on bearing characteristics, such as pressure distribution and load capacity, were investigated. This analysis showed that negative and positive pressures generated some clearance. Pressure distribution also changes in the gas supply hole number. A large load capacity was obtained at this clearance. To verify the phenomenon, measurement of pressure distribution was performed using experimental setup. Negative pressure could be confirmed actually in the large radial clearance condition.
\end{abstract}

Keywords: Ultra-High-Speed Rotary Machines, Hydroinertia Gas Journal Bearing, Pressure Distribution, Negative Pressure, Load Capacity

\section{Introduction}

Small externally pressurized gas journal bearings with diameters ranging between 1 and $6 \mathrm{~mm}$ have been implemented in ultra-high-speed rotational machines such as micro spinners for solid-state nuclear magnetic resonance (NMR) spectroscopy [1,2]. As shown in the inner configuration of the NMR instrument, these micro hydroinertia gas journal bearings were used to support the NMR spinner shaft, which was driven by an air turbine (Fig.1). An optical fiber detected the rotational speed. Resolution of the measurements can be improved by increasing the rotational speed. Therefore, gas lubricated bearings is practically used. These bearings had inherent orifice restrictors and required a larger radial clearance than conventional externally pressurized gas journal bearings. Inertial forces were dominant, generating a negative pressure on the bearing surface $[3,4,5]$. The large radial clearance resulted in stable operation of the high-speed rotating shaft because the reduced hydrodynamic effects suppressed the instabilities. In this readily feasible configuration, the $2 \mathrm{~mm}$ diameter shaft rotated at over $2,200,000 \mathrm{rpm}$ and the DN number, which corresponds to the product of the shaft diameter $(\mathrm{mm})$ and rotational speed (rpm), exceeded 4,400,000. The bearing not only NMR, it is also applicable to other ultra-high-speed small rotary machines as follows.

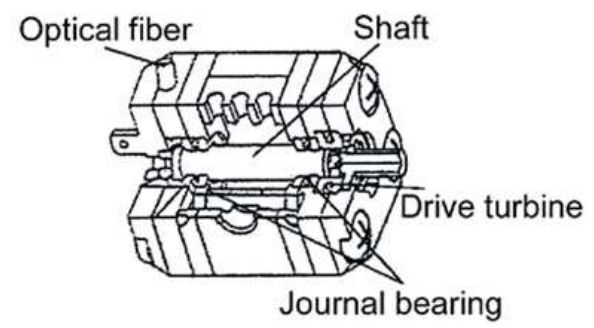

Figure 1. Inner configuration of the NMR device.

These bearings have also been used to study micromachines and palmtop gas turbine generators. 
Isomura et al. [6, 7] have constructed micromachines that rotated at $770,000 \mathrm{rpm}$ using $4 \mathrm{~mm}$ diameter journal bearings for a DN number of 3,080,000. Tanaka et al tested the utilization of these bearings in palmtop gas turbine generators and achieved a rotation of $890,000 \mathrm{rpm}$ using 4 $\mathrm{mm}$ diameter journal parts for the shaft for a DN number of $3,560,000[8,9]$. Waumans et al reached 1.2 million rpm (7.2 million DN) using aerodynamic journal bearing with a flexible support [10]. However, aerodynamic bearing needs small radial clearance, the radial clearance of $0.010 \mathrm{~mm}$ was used. In NMR device, large radial clearance bearing is suitable because assembly and disassembly of the shaft-bearing system are frequently repeated to change the sample. From this point of view, it is thought that use of hydroinertia bearings is desirable.

Hikichi et al have presented a method that provided the pressure distribution in micro hydroinertia gas journal bearings [4,5]. In this method, a circular thrust bearing surface replaced the journal surface for each supply hole. However, this approach allows a turbulent flow to become laminar, in contradiction with fluid dynamics laws, which dictate an increase in entropy. The effects of gas supply hole number and bearing clearance were not investigated. Yoshimoto et al also numerically and experimentally investigated the characteristics of the same circular thrust surface [11] and found that the calculations and experimental results agreed. However, none of these studies considered the overall journal bearing surface.

This study conducted a computational fluid dynamics (CFD) analysis of the entire journal bearing surface to elucidate the variation of the pressure distribution and static characteristics of bearings. This numerical approach was expected to fill this gap more effectively than experiments because the small bearing size made experimental verifications challenging. In addition, this approach was used to clarify the influence of shifting radial clearance and gas supply hole number on bearing characteristics. The target bearing diameter is $2 \mathrm{~mm}$. To verify the phenomenon, measurement of pressure distribution was performed using experimental setup. The bearing diameter is $10 \mathrm{~mm}$.

\section{Characteristics of Hydroinertia Gas Bearing}

Figure 2 compares schematic representations of conventional externally pressurized (Fig.2(a)) and hydroinertia gas journal bearings (Fig.2(b)). The supplied gas flows toward the bearing surface and generates pressure that supports the shaft. Since hydroinertia gas journal bearings present larger radial clearance than conventional bearings of the same circumference, the inertial force of the gas is dominant, producing supersonic gas flow, shock waves, and negative pressure. The large radial clearance has proven optimum for solid-state NMR because the shaft is frequently taken in and out of the spectrometer.
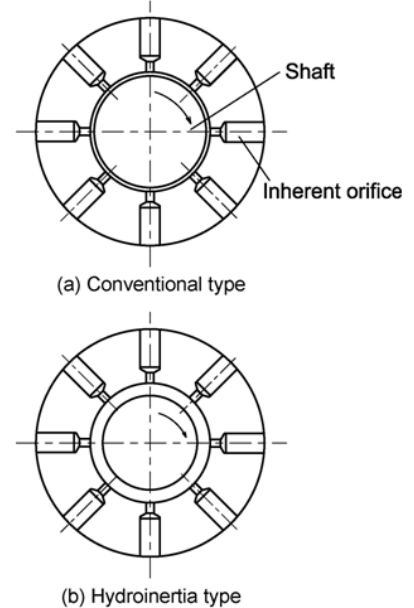

Figure 2. Configurations of conventional externally pressurized (a) and hydroinertia gas journal bearings (b).

\section{Validation of the CFD Analysis}

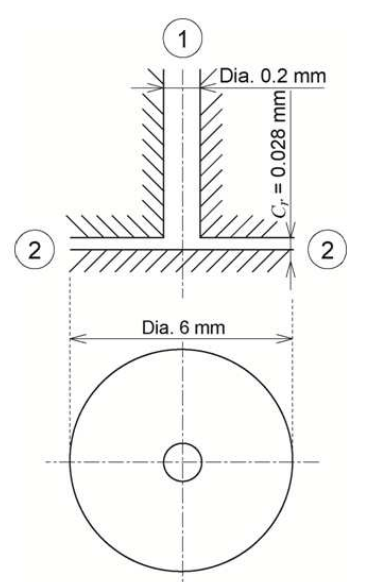

Figure 3. Hydroinertia thrust bearing model. Pressurized gas is supplied through the gas supply hole.

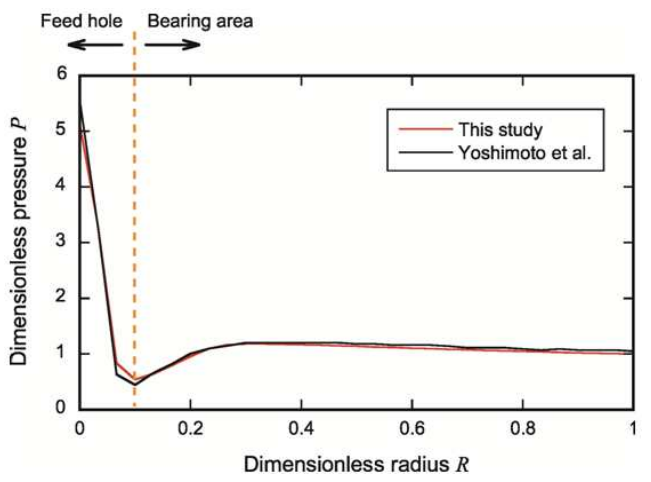

Figure 4. Analysis result of the hydroinertia thrust gas bearing.

The performance of hydroinertia gas journal bearing was determined using the general purpose CFD solver STAR $\mathrm{CCM}+$. The analytical model was built using a three-dimensional computer-aided design. Before the proposed bearing analysis was performed, computational accuracy was established by computing the pressure distribution of a hydroinertia thrust gas bearing with a 
single supply hole. These preliminary results were compared with data reported by Yoshimoto et al [11]. As shown in the representation of the thrust gas bearing model (Fig.3), pressurized gas is introduced through the gas supply hole (open circle 1, Fig.3) and flows out into the atmosphere (open circle 2) through the circular thrust bearing surface. Using the same specifications as Yoshimoto et al., the analysis model displayed an outer diameter $D$ of $6 \mathrm{~mm}$, a supply hole diameter $d$ of $0.2 \mathrm{~mm}$, and a clearance $C_{r}$ of $0.028 \mathrm{~mm}$. The dimensionless supply pressure $P$, defined as the ratio between supply gas $p_{s}$ and ambient pressures $p_{a}$, equaled 6 . Regarding the boundary conditions, the gas supply hole inlet and bearing surface outlet were set at $p_{s}$ and $p_{a}$, respectively. The computed pressure distribution is shown in Fig. 4 as a function of the dimensionless bearing radius $R$. An $R$ value of 0 represents the center of the bearing surface while a value of 1 corresponds to its edge. The distribution showed that the supplied gas pressure decreased to reach negative values at the outlet of the supply hole and subsequently increased to atmospheric pressure to recover a positive pressure. Small difference of the pressure was confirmed at outlet of the gas supply hole. This is thought that difference of the calculating models and grid types. Yoshimoto et al analyzed using two-dimensional analysis with structured grid. Whereas, this study analyzed using three-dimensional model with unstructured grid to fit the analysis model. From abovementioned reasons, it was thought that the validation of analysis accuracy was enough although the pressure distribution is different locally between the both analysis.

\section{CFD Analysis of the Hydroinertia Gas Journal Bearing}

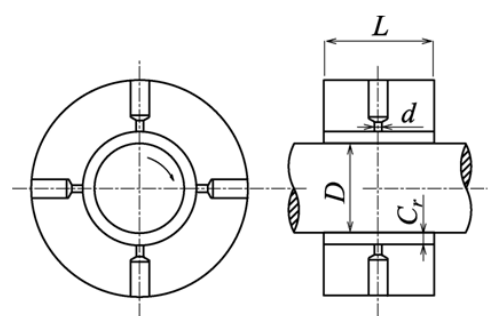

Figure 5. Analysis model of the hydroinertia thrust gas bearing.

Table 1. Bearing dimensions for analysis

\begin{tabular}{|c|c|c|c|c|c|}
\hline Type & Radial clearance $C_{r}$ & Feeding parameter $\Gamma$ & Gas supply hole number $n$ & Gas supply pressure $P$ & Eccentricity ratio $\varepsilon$ \\
\hline 1 & $0.0164 \mathrm{~mm}$ & 1.54 & 4 & \multirow{4}{*}{3} & \multirow{4}{*}{0.4} \\
\hline 2 & $0.0164 \mathrm{~mm}$ & 3.01 & 8 & & \\
\hline 3 & $0.0326 \mathrm{~mm}$ & 0.39 & 4 & & \\
\hline 4 & $0.0326 \mathrm{~mm}$ & 0.78 & 8 & & \\
\hline
\end{tabular}

The CFD analysis of the journal bearing surface was performed to elucidate the effects of the radial clearance and supply hole number on pressure distribution. The journal bearing model consisted of a single-row admission-type bearing (Fig.5) and resembled the practical NMR device. Conditions for the analysis are listed in Table 1. The analysis model displayed a bearing diameter $D$ of $2 \mathrm{~mm}$, a bearing length $L$ of $2 \mathrm{~mm}$, a gas supply hole diameter $d$ of $0.2 \mathrm{~mm}$, and dimensionless gas supply pressure $P=3$. The gas supply hole number $n$ equaled 4 or 8 . The radial clearance $C_{r}$ was set to 0.0164 or $0.0326 \mathrm{~mm}$ to achieve typical characteristics. The analysis was performed under static conditions, meaning that the rotor did not rotate. In addition, the rotor eccentricity ratio $\varepsilon$, which represents the ratio between the displacement of the shaft from bearing center and clearance, selected to 0.4 . This ratio is positive on the lower side of the bearing. Therefore, a $\varepsilon$ value of zero means that the rotor and bearing centers coincide. The dimensions and conditions of the analysis are listed in Table 1 for comparison purposes. In this study, non-dimensional parameter called as gas feeding parameter $\Gamma$ was used [12]. The $\Gamma$ is given by following formula:

$$
\Gamma=\frac{24 \mu n C_{f} r_{s} \sqrt{R T}}{p_{a} C_{r}{ }^{2}}
$$

where, the $\mu$ is gas viscosity the $k$ is number of gas supply hole, the $C_{f}$ is gas flow coefficient, $r_{s}$ is feed hole diameter, the $R$ is gas constant, the $T$ is gas temperature, the $p_{a}$ is atmospheric pressure and the $C_{r}$ is radial clearance of the baring. Similar flow resistance can be produced by matching this parameter although both dimensions are different. This values are also used in latter section to compare the pressure distribution with the experiments.

Figure 6(a) shows the pressure distribution of the overall bearing surface obtained for $C_{r}$ and $n$ values of $0.0164 \mathrm{~mm}$ and 4 , respectively (Type 1, Table 1). The shaft was moved downward, resulting in a small lower radial clearance (eccentric side) and a large clearance on the opposite side. On the eccentric side, the supplied pressure was maximum at the supply hole outlet and decreased monotonically to ambient pressure. The distribution showed that the pressure exhibited only positive values on the eccentric side whereas it attained negative values on the opposite side. This phenomenon produced simultaneous suction and repulsive forces. This means that restring force was large compared with the conventional externally pressurized gas journal bearings.

Figure 7 shows the pressure distribution of the overall bearing surface obtained for $C_{r}$ and $n$ values of $0.0164 \mathrm{~mm}$ and 8 , respectively (Type 2, Table 1 ). The numerical results showed that the pressure remained positive and supplied air did not reach supersonic speeds over the entire analyzed area. These positive pressures may originate from the 
increase in supply hole numbers which limits the space required for air acceleration in this Type 2 bearing compared to the Type 1 bearing. This positive pressure distribution is the same as for conventional externally pressurized gas journal bearings.

Figure 8 shows the distribution of the overall bearing surface obtained for $C_{r}$ and $n$ values of $0.0326 \mathrm{~mm}$ and 4, respectively (Type 3, Table 1). The numerical analysis indicated that the increased radial clearance on the eccentric side resulted in the negative pressure on eccentric and displacement sides. This observation suggests that this journal bearing exhibited a smaller load capacity than Type 1 bearing because the negative pressure produced a suction force in both sides.

Figure 9 shows the distribution of the overall bearing surface obtained for $C_{r}$ and $n$ values of $0.0326 \mathrm{~mm}$ and 8 , respectively (Type 4, Table 1). The numerical data revealed that increasing the supply hole number generated negative pressure on both sides of the shaft in a similar fashion as for Type 1 journal bearings.

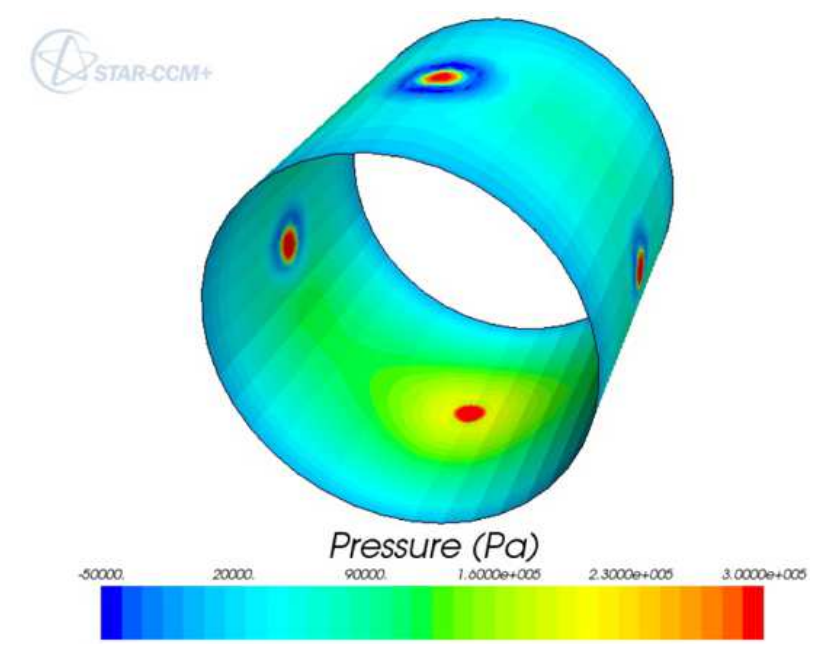

$\mathrm{C}_{\mathrm{r}}$ and $\mathrm{n}$ values equaled $0.0164 \mathrm{~mm}$ and 4 .

Figure 6. Pressure distribution of a Type 1 journal bearing model.

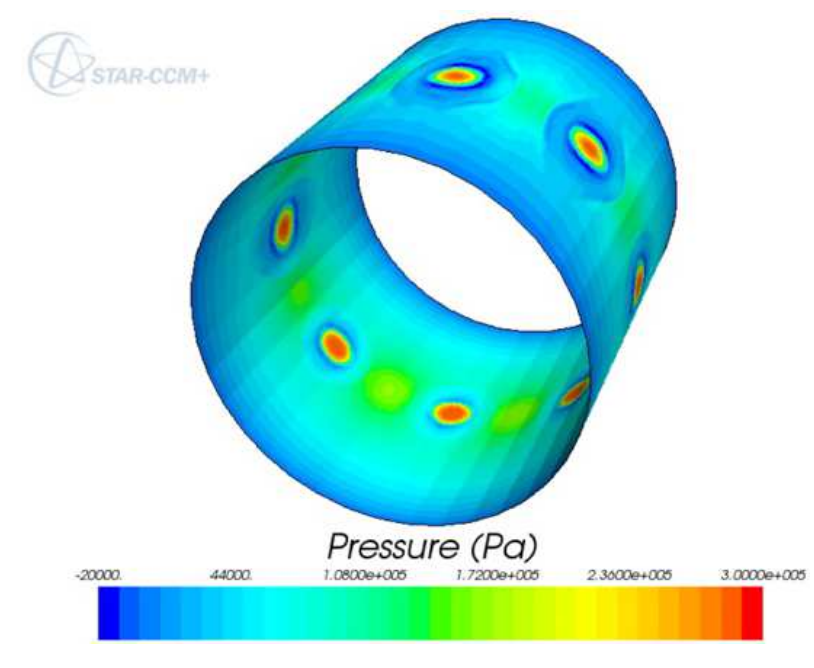

$\mathrm{C}_{\mathrm{r}}$ and $\mathrm{n}$ values equaled $0.0164 \mathrm{~mm}$ and 8 .

Figure 7. Pressure distribution of a Type 2 journal bearing model.

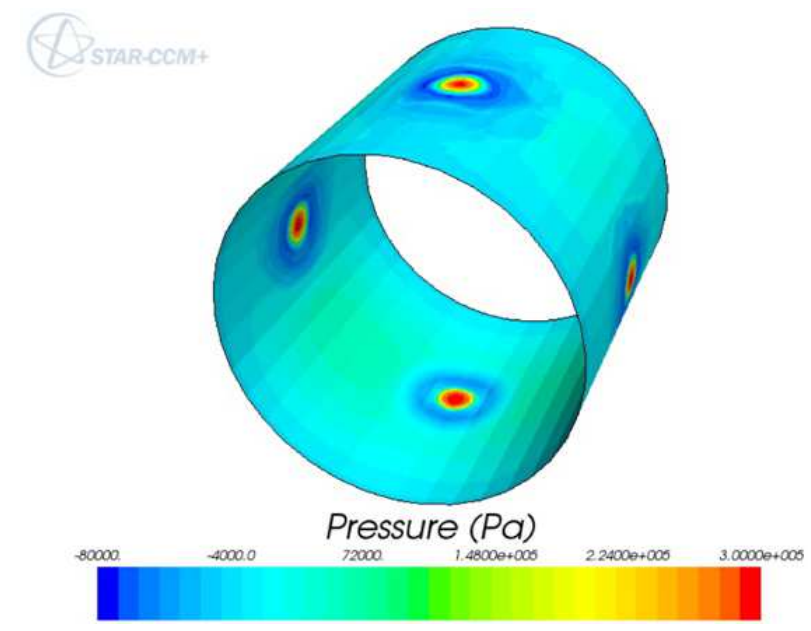

$\mathrm{C}_{\mathrm{r}}$ and $\mathrm{n}$ values equaled $0.0326 \mathrm{~mm}$ and 4 .

Figure 8. Pressure distribution of a Type 3 journal bearing model.
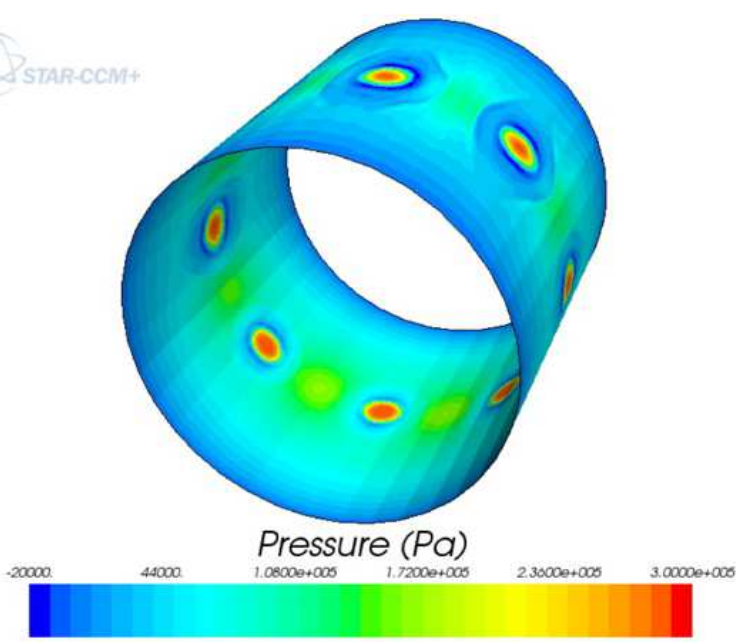

$\mathrm{C}_{\mathrm{r}}$ and $\mathrm{n}$ values equaled $0.0326 \mathrm{~mm}$ and 8 .

Figure 9. Pressure distribution of a Type 4 journal bearing model.

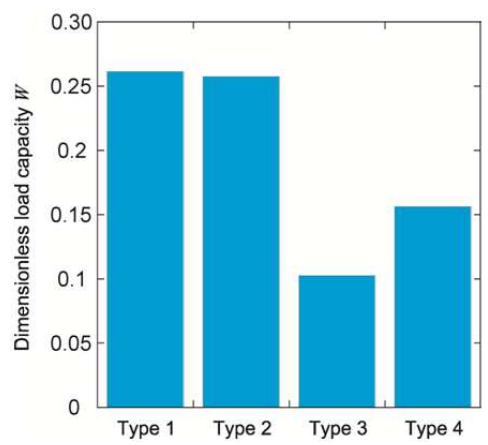

Figure 10. Load capacity of the bearings.

The static load capacity of journal gas bearings was calculated for various supply hole numbers and radial clearances by integrating pressure distributions over the entire bearing surface. This is obtained vertical direction of Fig.10. Figure 10 shows the dimensionless load capacity $W$ calculated at a $\varepsilon$ value of 0.4 for Type 1-4 bearings, in which the shaft was displaced toward the eccentric side. The dimensionless load capacity $W$ was expressed as 


$$
W=\frac{w}{L D\left(p_{s}-p_{a}\right)}
$$

where $w$ is the dimensional load capacity [N]. The numerical capacities agreed with values obtained using the conventional divergence formulation method for small clearance values $[13,14]$, suggesting that these results were highly reliable. The Types 1 and 2 bearings exhibited the largest load capacities, respectively. Particularly, Type 1 has large load capacity despite the $n=4$. This indicates that the generation of positive and negative pressures produces large $W$ values. The Type 4 bearing is third large load capacity, because it generated the similar pressure distribution as Type 1. It is thought that pressure was decreased because the clearance $C_{r}$ was increase. The Type 3 bearing showed the smallest load capacity because the generated pressure was also small and radial clearance was large.

\section{Measurement of the Pressure Distribution of the Bearings}

The pressure distribution of the bearing was measured using an experimental setup to qualitatively verify the results of the CFD analyses. This test was conducted using a $10 \mathrm{~mm}$ diameter bearing surface because the measurement using practical diameter $(2 \mathrm{~mm})$ is difficult at this time.

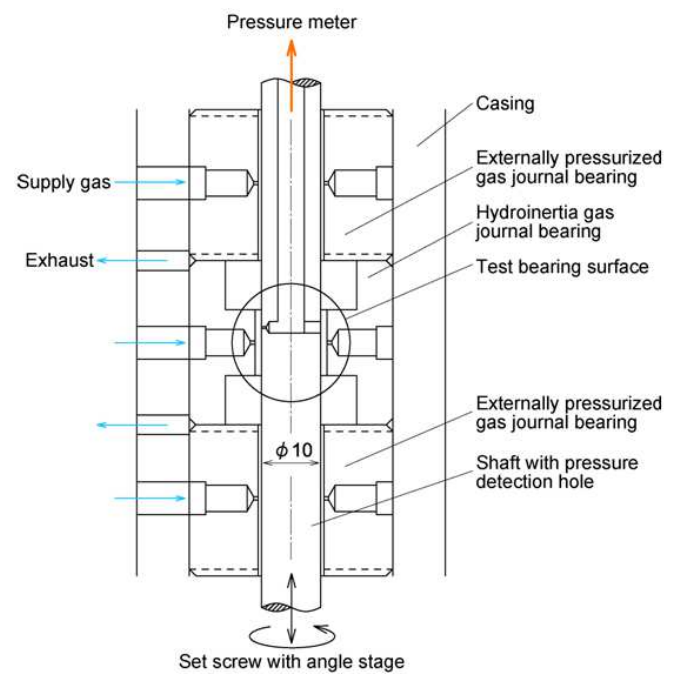

Figure 11. Experimental setup for the measuring pressure distribution.

The experimental setup is shown in Fig.11. The bearings and shaft are evaluated vertically to eliminate the effects of the shaft mass for the test bearing. The test hydroinertia bearing is installed in the casing. The manufactured actual clearance $C_{r}$ is 0.020 and $0.040 \mathrm{~mm}$, respectively. The supplied pressure is set at $P=6$. A shaft is installed in the bearing. The bearings have four gas feed holes. The $\Gamma$ of this bearing conditions are 1.54 for $C_{r}=0.020 \mathrm{~mm}$ and 0.39 for $C_{r}=0.040 \mathrm{~mm}$. These are same values with the CFD analysis. In this study, attempt to reveal the existence of negative pressure by $\Gamma$ by two conditions. A small orifice (diameter of $0.05 \mathrm{~mm}$ ) is set in this shaft for pressure measurement. This small diameter enables measurement of the static pressure only. The position of the shaft with the orifice is controlled by a set screw and an angle fine adjustment stage. The detected pressure is measured by a semiconductor-type pressure sensor. The shaft is supported by conventional externally pressurized gas journal bearings placed on both sides of the test hydroinertia bearing to position the center of the bearing, i.e., to ensure that $\varepsilon=0$. The radial clearance of the externally pressurized gas journal bearings is set to $0.01 \mathrm{~mm}$. Grooves are provided to ensure that atmospheric pressure is provided at the border of the bearings. The load capacity is sufficiently large to support the shaft. The axial and circumferential directions are measured at $0.1 \mathrm{~mm}$ and 5 degrees intervals, respectively.

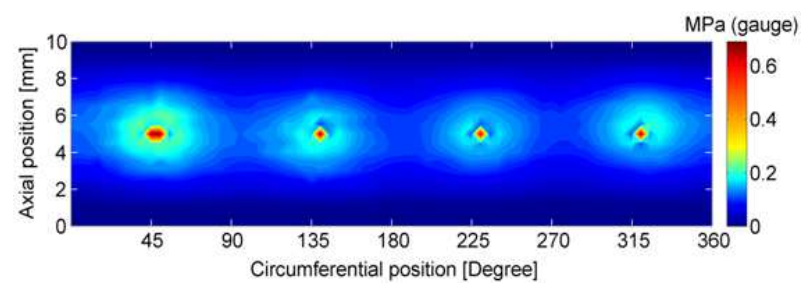

Figure 12. Pressure distribution of the bearing at $C_{r}=0.02 \mathrm{~mm}$.

Figure 12 shows the measured gauge pressure distributions of the test bearings for $C_{r}=0.02 \mathrm{~mm}$. Different pressure profiles are obtained depending on the gas supply hole considered. It is thought that the small eccentricity of the shaft in the bearing is the cause of the observed variation. An isotropic pressure distribution was obtained, and positive pressure was only confirmed near the supply hole. This distribution is same as commonly-used conventional externally pressurized gas journal bearings.

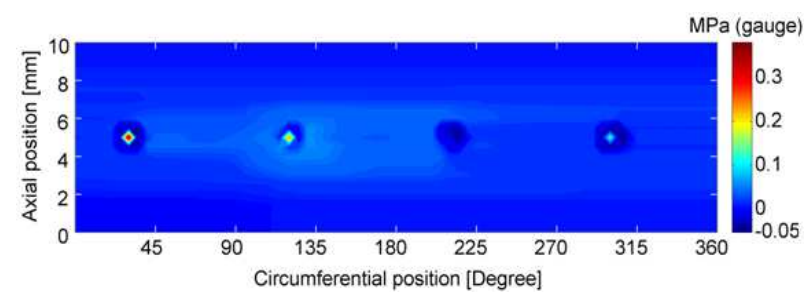

Figure 13. Pressure distribution of the bearing at $C_{r}=0.04 \mathrm{~mm}$.

Figure 13 shows the pressure distributions for $C_{r}=0.04$ $\mathrm{mm}$. Negative pressure distribution was obtained around the gas supply hole, that is similar to the results of the numerical analysis. Negative pressure was could be confirmed actually in the large radial clearance condition. The obtained measurements demonstrate the same tendencies as those exhibited by the numerical analysis, and qualitative agreement is confirmed. However, they are the result of specific conditions, the other experimental conditions are for further study. A detailed parameter study is most likely necessary to clarify the characteristics of this bearing, and this will be also carried out in the near future. 


\section{Conclusion}

In this study, a CFD analysis was performed to determine the detailed pressure distribution of micro hydroinertia gas journal bearings under static conditions using STAR CCM+. The generation of positive and negative pressures was observed for different bearing configurations. The occurrence of negative pressures was found to depend on the gas supply hole numbers regardless of the radial clearance. The load capacities of journal bearings were calculated for the analyzed configurations. Positive and negative pressure distributions generated large load capacities. These pressures are desirable in practical bearing applications since the bearings are small. To verify the phenomenon of the negative pressure generating, measurement of pressure distribution was performed using experimental setup. Negative pressure was could be confirmed actually in the large radial clearance condition. The same phenomenon was obtained experimentally; however, a parameter study is necessary to clarify the characteristics of this bearing, and this will be carried out in the near future. In addition, verification of the phenomena will be extended to rotating conditions in the near future.

\section{References}

[1] Y. Nishiyama, Y. Endo, T. Nemoto, H. Utsumi, K. Yamauchi, K. Hioka, T. Asakura, "Very fast magic angle spinning 1H-14N 2D solid-state NMR: Sub-micro-liter sample data collection in a few minutes," Journal of Magnetic Reso-nance, Vol. 208, Issue 1, 2011, pp. 44-48.

[2] K. Hioka, "Development of Ultra High-Speed 4 Million rpm Hydroinertia Gas Bearing for NMR Equipment." Journal of Japanese Society of Tribologists, Vol.56, No.3, 2011, pp. 143-148, (in Japanese).

[3] S. Tanaka, K. Hikichi. "Ultra High-Speed Gas Bearings for Ultra-Small Turbomachinery." Journal of Japanese Society of Tribologists, Vol.56, No.3, 2011, pp.137-142, (in Japanese).

[4] K. Hikichi, K. Shiratori, S. Togo, K. Hikoka, "Hydroinertia Gas Bearings and Their Application to High Speed Micro Spinners," Journal of the Japanese Society of Tribologists, Vol. 50, No. 6, 2005, pp. 465-470, (in Japanese).
[5] K. Hikichi, S. Goto, S. Togo, S. Tanaka, K. Isomura, "Hydroinertia gas bearings for micro spinners," Journal of Micromechanics and Microengineering, Vol. 15, 2005, pp. 228-232.

[6] K. Isomura, S. Togo, K. Hikichi, S. Goto, S. Tanaka, "Analytical and Experimental Study of Hydroinertia Gas Bearings for micromachine Gasturbines," Proceedings of ASME Turbo Expo 2005, 2005, No. GT2005-68401.

[7] K. Isomura, S. Tanaka, S. Togo, M. Esashi, "Development of high-speed micro-gas bearings for three-dimensional micro-turbo machines," Journal of Micromechanics and Microengineering, 15, 2005, S222-227.

[8] S. Tanaka, K. Hikichi, S. Togo, M. Esashi, "Turbo test rigwith hydroinertia air bearings for a palmtop gas turbine," Journal of Micromechanics and Microengineering, Vol. 14, 2004, pp. 1449-1454.

[9] S. Tanaka, M. Esashi, K. Isomura, K. Hikichi, Y. Endo, S.Togo, "Hydroinertia Gas Bearing System to Achieve $470 \mathrm{~m}$ per sec Tip Speed of 10 mm-Diameter Impellers," Transactions of ASME, Journal of Tribology, Vol. 129, 2007, pp. 655-659.

[10] T. Waumans, J. Peirs, F. Al-Bender, D. Reynaerts, "Aerodynamic journal bearing with a flexible, damped support operating at 7.2 million DN", Journal of Micromechanics and Microengineering, Vol.21, No,10, 2011, No.104014.

[11] S. Yoshimoto, M. Yamamoto, K. Toda, "Numerical Calculations of Pressure Distribution in the Bearing Clearance of Circular Aerostatic Thrust Bearings with a Single Air Supply Inlet," Transactions of ASME, Journal of Tribology, Vol. 129, Issue 2, 2007, pp. 384-390.

[12] H. Yabe, "A study on Run-out Characteristics of Externally pressurized Gas Journal Bearing (1st Report, Modified DF Method for Point-Source Solution)," Transactions of the Japan Society of Mechanical Engineers Series C, 1992, Vol. 58, No. 548, pp. 1170-1176 (in Japanese with English abstract).

[13] S. Togo, Gas Bearing Design Guide Book, 2002, Kyoritsu Shuppan Co., Ltd. (in Japanese).

[14] S. Togo, I. Kawashima, T. Ise, K. Hikichi, Y. Endo, T. Maeda, Gas Bearing - Technics of design and operation and production-, 2014, Kyoritsu Shuppan Co., Ltd. (in Japanese). 